\title{
Impact of lgt mutation on lipoprotein biosynthesis and in vitro phenotypes of Streptococcus agalactiae
}

\author{
Correspondence \\ Dean J. Harrington \\ d.harrington@bradford.ac.uk
}

Received 20 October 2008

Revised 25 February 2009

Accepted 26 February 2009

\author{
Beverley A. Bray, ${ }^{1}$ lain C. Sutcliffe ${ }^{2}$ and Dean J. Harrington ${ }^{1}$ \\ ${ }^{1}$ University of Bradford, West Yorkshire BD7 1DP, UK \\ ${ }^{2}$ Northumbria University, Newcastle upon Tyne NE1 8ST, UK
}

\begin{abstract}
Although Streptococcus agalactiae, the group B Streptococcus, is a leading cause of invasive neonatal disease worldwide the molecular basis of its virulence is still poorly understood. To investigate the role of lipoproteins in the physiology and interaction of this pathogen with host cells, we generated a mutant S. agalactiae strain (A909 L Lgt) deficient in the Lgt enzyme and thus unable to lipidate lipoprotein precursors (pro-lipoproteins). The loss of pro-lipoprotein lipidation did not affect the viability of $S$. agalactiae or its growth in several different media, including cationdepleted media. The processing of two well-characterized lipoproteins, but not a non-lipoprotein, was clearly shown to be aberrant in $\mathrm{A} 909 \Delta \mathrm{Lgt}$. The mutant strain was shown to be more sensitive to oxidative stress in vitro although the molecular basis of this increased sensitivity was not apparent. The inactivation of Lgt also resulted in changes to the bacterial cell envelope, as demonstrated by reduced retention of both the group $B$ carbohydrate and the polysaccharide capsule and a statistically significant reduction $(P=0.0079)$ in $\mathrm{A} 909 \Delta \mathrm{Lgt}$ adherence to human endothelial cells of fetal origin. These data confirm that failure to process lipoproteins correctly has pleiotropic effects that may be of significance to $S$. agalactiae colonization and pathogenesis.
\end{abstract}

\section{INTRODUCTION}

Streptococcus agalactiae, the group B Streptococcus (GBS), is a leading cause of invasive neonatal disease worldwide (Henneke \& Berner, 2006; Johri et al. 2006). Although prenatal screening and intra-partum antibiotic prophylaxis has been successful in reducing the burden of GBS disease, significant levels of GBS disease remain. For example, in the UK its incidence is 0.72 cases per 1000 live births (Heath et al., 2004). Consequently there is a need for greater understanding of the molecular basis of GBS pathogenicity in order to inform novel strategies for prophylaxis (notably vaccine development) and/or therapy in several different population groups. Such studies should be greatly facilitated by the availability of three complete and five draft genome sequences for GBS strains (Tettelin et al., 2002, 2005; Glaser et al., 2002).

Bacterial lipoproteins (Lpp) are an important family of proteins that are anchored to cell membranes, where they carry out diverse functions (Braun \& Wu, 1994; Sutcliffe \& Russell, 1995; Sutcliffe \& Harrington, 2002; Hutchings

Abbreviations: GBS, group B Streptococcus; HUVEC-C, human umbilical vein endothelial cell line; Lgt, prolipoprotein diacylglyceryl transferase; Lpp, lipoproteins.

A supplementary figure showing the strategy for generating allelic replacement mutants of $S$. agalactiae A909 is available with the online version of this paper. et al., 2009). Lipoprotein biosynthesis depends on the synthesis and translocation of precursor Lpp (prolipoproteins; pro-Lpp) with type II signal peptides. Following translocation, typically by the Sec pathway, pro-Lpp are lipid modified by addition of diacylglyceride to a cysteine residue within the Lpp signal peptide, a reaction carried out by the prolipoprotein diacylglyceryl transferase (Lgt; Braun \& Wu, 1994; Hutchings et al., 2009). The signal peptide is then removed by a lipoprotein-specific signal peptidase (Lsp; Braun \& Wu, 1994; Hutchings et al., 2009), leaving the lipid-modified cysteine as the $\mathrm{N}$-terminus of the mature lipoprotein. These two steps are apparently sufficient for anchoring of Lpp to the extracytoplasmic face of the plasma membrane in Gram-positive bacteria (Sutcliffe \& Harrington, 2002; Hutchings et al., 2009).

The conserved features of Lpp signal peptides have allowed the development of bioinformatic strategies for the identification of putative Lpp encoded in bacterial genomes (Sutcliffe \& Harrington, 2002; Rahman et al., 2008). We have previously analysed the genomes of two GBS strains (Sutcliffe \& Harrington, 2004) and determined that putative Lpp represent about $2 \%$ of the GBS proteome. Many of these putative Lpp have predicted roles that are likely to affect the ability of GBS to survive in vivo. For example, more than 20 solute-binding proteins for the import of diverse substrates by $\mathrm{ABC}$ transport systems have been identified (Sutcliffe \& Harrington, 
2004). In addition, some putative Lpp, including the previously characterized adhesin Lmb (Spellerberg et al., 1999; Tenenbaum et al., 2007), may have direct roles in host colonization. Moreover, Lpp are apparently crucial to the recognition of GBS by Toll-like receptor 2 (Henneke et al. 2008).

As Lpp are likely to make an important contribution to the physiology and virulence of GBS we have investigated Lpp biosynthesis in this important pathogen.

\section{METHODS}

Bacterial strains and growth media. S. agalactiae A909 and its derivatives were cultured aerobically in Todd-Hewitt Broth (THB; Oxoid) or anaerobically on Granada medium (Biomedics) at $37{ }^{\circ} \mathrm{C}$. For some experiments, media with depleted metal ion content were prepared by incubating THB with Chelex-100 resin as described previously (Bray et al., 2009). Antibiotics were added to growth media as appropriate to allow selection of antibiotic-resistant GBS strains.

Generation of Lgt-deficient (A909 $\Delta$ Lgt) and capsule-deficient (A909 $\Delta$ CPSE) strains of S. agalactiae A909 by allelic replacement. Lgt-deficient and CPSE-deficient mutants of S. agalactiae A909 were generated by allelic replacement. Copies of the lgt and $c p s E$ genes containing in-frame deletions were constructed by PCR using primers based on the A909 genome sequence (Table 1; see also Supplementary Fig. S1, available with the online version of this paper). For the $l g t$ gene these primer pairs amplified $5^{\prime}$ and $3^{\prime}$ DNA fragments, leaving $354 \mathrm{bp}$ of the central portion of the lgt gene unamplified. For the cpsE primers, these primer pairs amplified $5^{\prime}$ and $3^{\prime}$ DNA fragments, leaving $741 \mathrm{bp}$ of the cpsE gene unamplified. To combine the upstream and downstream fragments, one primer of each downstream primer pair (SALGTTOM3U/CPSEBEV3U) contained a $5^{\prime}$ sequence which was complementary to the $3^{\prime}$ sequence of one of the upstream primers (SALGTTOM2L/CPSEBEV2L). The terminal primers were also engineered to contain cleavage sites for ApaI and SacII restriction enzymes.

First-round PCR fragments were amplified using chromosomal A909 DNA as template and Pfu DNA polymerase. These DNA fragments were purified and combined to serve as the template for the second round of PCR, using only the terminal primers (SALGTTOM1U/ SALGTTOM4L or CPSEBEV1U/CPSEBEV4L) in order to produce a single contiguous DNA fragment for each gene, each of which contained an in-frame deletion. Second-round PCR products were Atailed with Taq polymerase and ligated into the pGEM-T cloning vector (Promega). The cloned fragments were then excised from the resulting recombinant pGEM-T vectors, using ApaI and SacII, and subcloned into $\mathrm{pG}^{+}$host9: ISS 1 (Maguin et al., 1996) to generate the plasmids $\mathrm{pBB} \Delta$ Lgt and $\mathrm{pBB} \Delta \mathrm{CPSE}$.

Transformants containing either pBB $\Delta$ Lgt or pBB $\Delta$ CPSE were subjected to two rounds of homologous recombination as previously described (Biswas et al., 1993; Maguin et al., 1996). The presence of mutant alleles in the chromosome of putative mutants was determined by amplifying the allele present (using $P f u$ DNA polymerase) and sequencing the PCR products.

Radiolabelling of lipoproteins using $\left[{ }^{14} \mathrm{C}\right]$ palmitic acid. Radiolabelling was carried out as described by Stoll et al. (2005). Briefly, $\left[{ }^{14} \mathrm{C}\right]$ palmitic acid $\left(3.32 \mu \mathrm{Ci} \mathrm{ml}{ }^{-1} ; 123 \mathrm{kBq} \mathrm{ml}^{-1}\right)$ was added to early-exponential-phase GBS cultures and growth continued until the cultures reached late exponential phase. Cells were harvested and washed three times in PBS. Soluble cell extracts were separated on $12 \%$ SDS-polyacrylamide gels. After Coomassie blue staining, gels were soaked in Amplify solution (Amersham) for $30 \mathrm{~min}$ before being dried. Dried gels were exposed to Kodak X-OMAT LS X-ray film $\left(-80{ }^{\circ} \mathrm{C}, 2\right.$ weeks) before being developed.

Investigation of lipoprotein processing in S. agalactiae strains. Overnight cultures of GBS strains were diluted 1 in 20 in fresh THB and grown to mid-exponential phase. Cells were harvested and cell fractions were separated as previously described (Low et al., 2003). Briefly, cell pellets were washed thoroughly in PBS, resuspended in

Table 1. Oligonucleotide primers and plasmids used in this study

\begin{tabular}{|c|c|c|}
\hline Primer or plasmid & Sequence $\left(5^{\prime}-3^{\prime}\right)$ or description & Reference \\
\hline \multicolumn{3}{|l|}{ Primers $^{\star}$} \\
\hline SALGTTOM1U & GGGCCCTTGATTATTATGCACCAGAGCG & \\
\hline SALGTTOM2L & 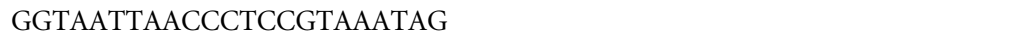 & \\
\hline SALGTTOM3U & СTATTTACGGAGGGTTAATTACCGAAGGGATGAGGACAGATAGC & \\
\hline SALGTTOM4L & CCGCGGTTCTTACTAGCGATGCTGGC & \\
\hline CPSEBEV1U & GGGCCCCTTGCAATTACCTCTGTTAGGG & \\
\hline CPSEBEV2L & GTCAAACTTGCAGAAAAATAAACC & \\
\hline CPSEBEV3U & GGTTTATTTTTCTGCAAGTTTGACCACGGGTGCTATTATAGGTTTG & \\
\hline CPSEBEV4L & CCGCGGCTCCTAGCATCTTCTTTATCAAAG & \\
\hline \multicolumn{3}{|l|}{ Plasmids } \\
\hline $\mathrm{pG}^{+}$host9: ISS1 & Replication-thermosensitive derivative of pWV01 containing cloned ISS1 sequence & Maguin et al. (1996) \\
\hline $\mathrm{pBB} \Delta \operatorname{lgt}$ & $\mathrm{pG}^{+}$host9: ISS1† containing in-frame deleted lgt gene of $S$. agalactiae & This study \\
\hline $\mathrm{pBB} \Delta \mathrm{cpsE}$ & $\mathrm{pG}^{+}$host9: ISS $1 \dagger$ containing in-frame deleted $c p s E$ gene of $S$. agalactiae & This study \\
\hline pGEM-T & T-A cloning vector & Promega \\
\hline
\end{tabular}

*Underlined sequences represent engineered restriction sites for ApaI (GGGCCC) and SacII (CCGCGG). The italicized sequences in SALGTTOM3U and CPSEBEV3U are the reverse and complementary sequences to the SALGTTOM2L and CPSEBEV2L primer sequences, respectively, and provide the overlapping sequences for the overlap-deletion PCR strategy.

$\dagger$ The ISS1 sequence is completely removed during the cloning strategy. 
$200 \mu$ lysozyme solution $\left(0.2 \mathrm{mg} \mathrm{ml}^{-1}\right.$ in $\left.\mathrm{PBS}\right)$ and incubated at $37{ }^{\circ} \mathrm{C}$ for $20 \mathrm{~min}$. The suspensions were then vortexed with $200 \mathrm{mg}$ glass beads (acid washed, 425-600 $\mu \mathrm{m}$; Sigma) for $2 \mathrm{~min}$. The beads were allowed to settle under gravity and the supernatant centrifuged $(800 \mathrm{~g}, 1 \mathrm{~min})$ to pellet any unlysed cells. The supernatant was recovered and centrifuged $\left(13000 \mathrm{~g}, 30 \mathrm{~min}, 4{ }^{\circ} \mathrm{C}\right)$ to generate a lower, membrane-enriched fraction and an upper phase which contained the cytoplasmic and lysozyme-released (i.e. cell-wallassociated) fractions. Fractions were analysed on $10 \%$ SDS-polyacrylamide gels and stained with Coomassie blue or Western blotted as described by Hamilton et al. (2000). Blotted membranes were probed with anti-MtuA antibody (Smith et al., 2003; diluted 1/1000), anti-SAG0808 antibody (Tettelin et al., 2002; diluted 1/1000) or antiSip antibody (Brodeur et al., 2000; diluted 1/500).

Sensitivity of S. agalactiae strains to oxidative stress and aminopterin. The sensitivity of GBS strains to challenge with hydrogen peroxide was assessed using the method of Johnston et al. (2004). Manganese co-factored superoxide dismutase activity was assessed by the zymographic method of Janulczyk et al. (2003). Aminopterin was added to mid- to late-exponential-phase GBS cultures at final concentrations of $0,0.1,1.0,10$ or $100 \mathrm{nM}$. The $\mathrm{OD}_{600}$ of each culture was taken immediately pre- and post-addition. The cultures were incubated at $37{ }^{\circ} \mathrm{C}$ for a further $2 \mathrm{~h}$ and the $\mathrm{OD}_{600}$ monitored. All experiments were performed in triplicate.

Quantification of streptococcal Lancefield group B carbohydrate by latex agglutination. A Streptococcal Latex Grouping kit (Oxoid) was used, following the manufacturer's instructions. Standardized aliquots of washed GBS cells were resuspended in $200 \mu \mathrm{l}$ extraction buffer (Oxoid). After incubation at $37^{\circ} \mathrm{C}$ for $10 \mathrm{~min}$ the extract was serially diluted and $5 \mu \mathrm{l}$ of the dilutions was mixed with $20 \mu \mathrm{l}$ latex bead suspension on non-absorbent card. The time taken for visible agglutination to start to occur was recorded. All experiments were performed in triplicate.

Investigation of capsular polysaccharide expression. Capsular polysaccharide in GBS strains was detected with rabbit anti-CPS (Ia) antiserum, using the semiquantitative method of Chaffin et al. (2000).

Gold labelling of GBS strains was carried out using the protocol of Dixon et al. (2001). Rabbit anti-CPS (Ia) antiserum was used as primary antibody and goat anti-rabbit IgG conjugated with $10 \mathrm{~nm}$ colloidal gold particles (Sigma-Aldrich; diluted 1/10 in PBS-Tween) as secondary antibody.

\footnotetext{
Adherence to and invasion of the immortalized Human Umbilical Vein Endothelial Cell cell line (HUVEC-C) by $\boldsymbol{S}$. agalactiae strains. HUVEC-C cells (ATCC; CRL-1730) were grown in Medium 199 (Sigma, UK) with $10 \%$ (v/v) fetal calf serum at $37{ }^{\circ} \mathrm{C}$ in a $5 \% \mathrm{CO}_{2}$ atmosphere. Prior to adherence/invasion experiments being carried out, HUVEC-C cells were seeded into 12-well plates and grown to confluence (approx. $2 \times 10^{5}$ cells per well) as determined by visual inspection using an inverted microscope. Adhesion and invasion were determined by the method of Rubens et al. (1992). Overnight GBS cultures were diluted into fresh THB and incubated at $37{ }^{\circ} \mathrm{C}$ to mid-exponential phase. Standardized aliquots of cells were harvested, washed three times in sterile PBS and resuspended in cell culture growth medium without antibiotics to give a cell density of $2 \times 10^{5}$ c.f.u. $\mathrm{ml}^{-1}$. Triplicate wells containing confluent HUVEC-C cells were inoculated with $2 \times 10^{5}$ c.f.u. of bacterial cell suspensions (i.e. a $1: 1$ ratio of bacteria to HUVEC-C cells) in duplicate 12 -well plates. The plates were centrifuged $(800 \mathrm{~g}, 10 \mathrm{~min})$ to bring the bacteria into contact with the HUVEC-C monolayers and then incubated for $2 \mathrm{~h}$ at $37{ }^{\circ} \mathrm{C}$ in a $5 \% \mathrm{CO}_{2}$ atmosphere. The culture medium was removed from the wells and the HUVEC-C cells were washed three times with sterile PBS. To measure bacterial adhesion
}

plus invasion the HUVEC-C cells from the first plate were detached by the addition of $0.4 \mathrm{ml}$ trypsin-EDTA solution and incubation at $37{ }^{\circ} \mathrm{C}$ for $4 \mathrm{~min}$ before being gently resuspended. The detached cells were vortexed for $1 \mathrm{~min}$ to ensure complete disruption of HUVEC-C clumps and $1.2 \mathrm{ml} 0.025 \%(\mathrm{w} / \mathrm{v})$ Triton X-100 was added to lyse the HUVEC-C cells before further vortexing. Each suspension was serially diluted, plated in triplicate onto Todd-Hewitt agar and incubated overnight at $37^{\circ} \mathrm{C}$ to determine bacterial counts. To measure bacterial invasion, $1 \mathrm{ml}$ culture medium containing $5 \mu \mathrm{g}$ penicillin $\mathrm{G}$ and $100 \mu \mathrm{g}$ gentamicin was added to the wells of the duplicate 12well plate and incubated for $2 \mathrm{~h}$ at $37{ }^{\circ} \mathrm{C}$ to kill any extracellular bacteria. HUVEC-C monolayers were then washed, detached, lysed and plated on agar as described above. Bacterial colony counts were used to calculate the mean number of bacteria that had invaded the HUVEC-C cells for each strain. This number was then subtracted from the mean colony counts obtained for the equivalent wells in the corresponding adherence plus invasion assay to give a mean number of bacteria that had adhered to the HUVEC-C cells for each strain. Each mean was expressed as a percentage of the original bacterial inoculum added to each HUVEC-C monolayer.

\section{RESULTS AND DISCUSSION}

\section{Construction of an Lgt-deficient strain of GBS A909}

Bioinformatic analysis of the GBS genomes identified a single candidate Lgt protein (PFAM family PF01790), annotated as SAK_0863 in the genome of strain A909, which exhibits 123/254 (48\%) amino acid sequence identity to the characterized Lgt of Bacillus subtilis (Leskelä et al., 1999). An overlap-deletion PCR strategy was used to construct an in-frame $354 \mathrm{nt}$ deletion within lgt and an unmarked A909 strain carrying a mutant lgt allele (strain A909 $\Delta$ Lgt) was obtained by allelic replacement (Fig. S1). The deletion within lgt was confirmed by amplification of an appropriately truncated PCR product from A909ALgt in comparison to the wild-type A909 and DNA sequencing of the truncated amplification product (data not shown). This deletion removes a section of SAK_0863 (from $\mathrm{Gly}_{97}$ to $\mathrm{Ile}_{214}$ ) which contains many of the conserved residues predicted to be needed for Lgt function (our unpublished observations) and should result in the production of a truncated Lgt protein with a significantly altered topology. The generation of a viable Lgt-deficient GBS mutant is consistent with previous reports indicating that loss of Lgt does not significantly affect in vitro viability of Gram-positive bacteria (Leskelä et al., 1999; Petit et al., 2001; Stoll et al. 2005; Hamilton et al., 2006; Baumgärtner et al., 2007), including GBS (Henneke et al., 2008).

To verify that Lpp biosynthesis had been abrogated in A909 $\Delta$ Lgt, the parent and mutant strains were grown in the presence of $\left[{ }^{14} \mathrm{C}\right]$ palmitic acid. Extracted proteins were separated by SDS-PAGE and analysed by autoradiography (Fig. 1). As expected, multiple selectively radiolabelled Lpp bands, predominantly within the $25-37 \mathrm{kDa}$ size range, were clearly detected in the parent strain whereas these bands were not evident in the extracts from strain 


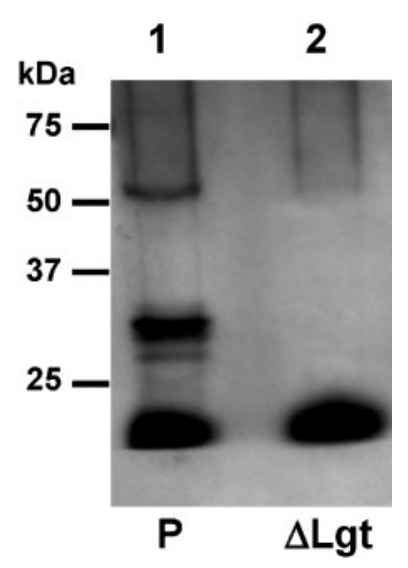

Fig. 1. Lipoprotein profiles of S. agalactiae A909 and A909 L Lgt. Lipoproteins in GBS strains were labelled by the addition of $\left[{ }^{14} \mathrm{C}\right]$ palmitic acid to the growth medium. Labelled proteins were separated by SDS-PAGE and visualized by autoradiography. Lane 1, parent strain $(P)$; lane 2, A909 $\Delta$ Lgt $(\Delta \mathrm{Lgt})$. Radiolabelled palmitic acid incorporated in membrane lipids can be seen at the bottom of each lane.

A909 $\Delta$ Lgt. Thus, direct biochemical evidence was obtained to support the prediction that SAK_0863 is the GBS Lgt protein and that this activity had been successfully inactivated in strain A909 $\Delta$ Lgt. This phenotype is identical to that of a recently described GBS strain NEM316 lgt deletion mutant (Henneke et al. 2008).

\section{Effect of Lgt inactivation on lipoprotein processing}

Western blotting was used to characterize the effects of lgt mutation on specific lipoproteins (Fig. 2). MtsA (SAK_1556) is an experimentally verified Lpp in GBS (Bray et al., 2009; Henneke et al., 2008). Cellular fractions were prepared from strains A909 and A909 LLgt and analysed by Western blotting with a polyclonal antibody that cross-reacts with MtsA (Bray et al., 2009). In the parent strain a single cross-reacting band of $\sim 34 \mathrm{kDa}$ was seen in all cellular fractions (Fig. 2a, lanes 2, 4 and 6). In contrast, the failure to lipidate MtsA in strain A909 $\Delta$ Lgt resulted in the appearance of two cross-reacting bands in each fraction (Fig. 2a, lanes 3, 5 and 7). One of the two bands appeared to be about the same molecular mass as the mature protein observed in the parent strain ('mature-like' protein) whilst the higher molecular mass band is presumed to be a non-lipidated MtsA pro-Lpp with an uncleaved signal peptide. The 'mature-like' protein probably represents non-lipidated pro-Lpp which has been proteolytically processed by cleavage of some or all of its lipoprotein signal peptide. 'Mature-like' products from Lpp precursors have been observed previously in lgt mutants (Bubeck Wardenburg et al. 2006; Hamilton et al., 2006), and in some Gram-positive bacteria Lsp can process both lipidated and unlipidated Lpp precursors (Baumgärtner et al., 2007; Henneke et al., 2008; Denham et al., 2009). Almost identical results were obtained for the putative maturase lipoprotein SAK_0932 (Fig. 2b). In both cases additional cross-reacting bands of $50-75 \mathrm{kDa}$ were seen in the membrane-enriched and cell-wall-associated

(a)

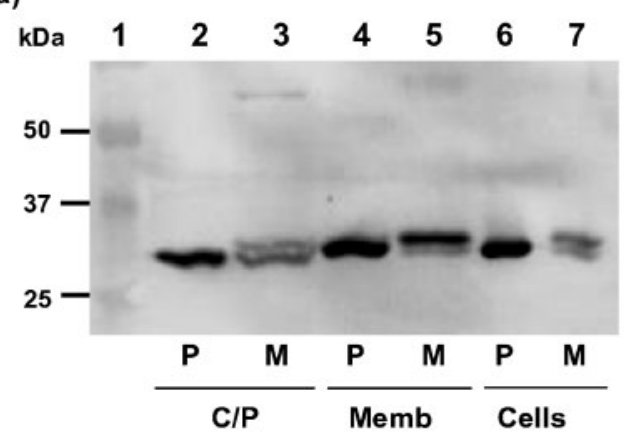

(b)

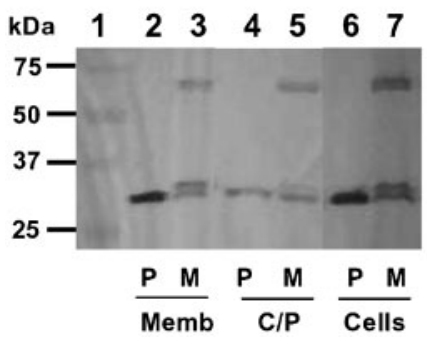

(c)

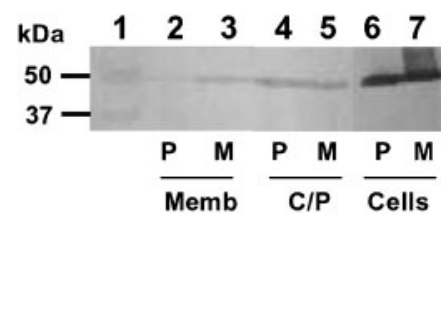

Fig. 2. Aberrant processing of lipoproteins in S. agalactiae A909 $\Delta$ Lgt. Localization of the MtsA lipoprotein (a), the putative maturase lipoprotein (b) and the non-lipoprotein Sip protein (c) was investigated by Western blotting with specific antisera. In each panel, lane 1 contains molecular mass markers and other lanes contain proteins recovered from the cytoplasmic/peptidoglycan fraction (C/P), the membrane-enriched fraction (Memb) and the whole-cell proteins (Cells) as indicated. Lanes 2, 4 and 6 contain proteins extracted from the A909 parent strain (P). Lanes 3, 5 and 7 contain proteins extracted from the A909 $\Delta$ Lgt mutant (M). All sample lanes contain $50 \mu \mathrm{g}$ total protein. 
fractions of the Lgt mutant but not the parent strain (Fig. $2 \mathrm{~b}$, lanes 3 and 5). As these bands were detected with specific antibodies in the mutant strain only it is possible that they represent aggregated forms of the pro-Lpp. There were no differences in the processing of the nonlipoprotein Sip (Brodeur et al., 2000) in the A909 $\Delta$ Lgt mutant (Fig. 2c), confirming that aberrant processing is restricted to lipoproteins.

The data reported here for MtsA processing are generally consistent with the observations of Henneke et al. (2008) for the same protein (ScaA) in an Lgt-deficient mutant of GBS strain NEM316. While those authors did not observe the build-up of the higher molecular mass form of ScaA (which we attribute to the non-lipidated pro-Lpp), a cellassociated 'mature-like' ScaA protein was detected in their lgt mutant. Moreover ScaA with a mature $\mathrm{N}$-terminus was detected in culture supernatants of the mutant, suggesting functional Lsp activity in the absence of lipidation. Henneke et al. (2008) also demonstrated that ScaA was further processed by a distinct signal peptidase activity and released into culture supernatants of an lgt/lsp double mutant. Similarly, the Eep peptidase has been shown to act on lipoprotein precursors in Streptococcus uberis (Denham et al., 2008). In contrast to their results for ScaA, and our results for MtsA and SAK_0932, Henneke et al. (2008) did not detect any cell-associated Lmb in their lgt mutant but did show that a form of Lmb was released into culture supernatants. Evidently, inactivation of Lpp biosynthesis results in aberrant processing and possibly mislocalization of Lpp precursors, the precise nature of which can vary between different Lpp in the same strain and between the same Lpp in different strains.

\section{Phenotypic characterization of A909 $\Delta$ Lgt}

Strain A909 $\Delta$ Lgt exhibited no differences in its general growth characteristics (colony morphology; haemolytic capacity on Columbia blood agar; pigmentation on Granada medium). The fatty acid profile of the whole cells of strain A909 $\mathrm{Lgt}$ was also the same as that of the parent strain (data not shown), suggesting that the failure to turn over membrane lipids into lipoproteins does not significantly affect the cell membrane composition.

Growth curves for A909 $\Delta$ Lgt in unbuffered THB at $\mathrm{pH} 7$ and pH 5 and in media depleted of divalent cations by Chelex treatment were also not significantly different from those of the parent strain (data not shown). These results are in good agreement with the data of Henneke et al. (2008), who reported that deletion mutants of lgt, lsp and lgt/lsp in GBS strain NEM316 exhibited normal growth in nutrient-rich media and only minor impairment of growth rates in nutrient-poor or minimal media. In contrast, the growth of an Lgt mutant of Staphylococcus aureus was severely impaired in nutrient-poor (iron-depleted) medium (Stoll et al., 2005).

Amino acids are one of the primary groups of substrates transported by putative lipoproteins in their role as solute- binding proteins in $\mathrm{ABC}$ transporter systems (Sutcliffe \& Harrington, 2002, 2004). If the ability to transport amino acids was impaired in the A909 $\Delta$ Lgt mutant, then it might be evident by a decrease in the sensitivity of the bacteria to the toxic amino acid analogue aminopterin. However, at doses of $0.5,5$ and $50 \mathrm{nM}$ aminopterin no effect on growth could be seen in either strain (data not shown), suggesting that this amino acid analogue is not imported by either strain.

We assayed sensitivity of the GBS wild-type and A909 $\Delta$ Lgt strains to hydrogen peroxide exposure. The Lgt mutant was significantly more sensitive to oxidative stress in a timeand dose-dependent manner (Fig. 3). Further studies will be needed to establish the basis for this phenotype but it was interesting to note that zymographic analysis revealed no obvious change in the level of the superoxide dismutase activity in strain A909ALgt compared with the parent strain (Fig. 3, inset). Since subsequent experiments suggested that the Lgt mutant may have altered levels of encapsulation we also tested the sensitivity of a capsuledeficient mutant (A909LCPSE) to hydrogen peroxide exposure. There was no significant difference in the sensitivity of A909 $\triangle$ CPSE and the parent strain at any of the hydrogen peroxide doses tested (data not shown).

\section{Effect of Lgt mutation on cell envelope properties of GBS}

GBS strains display both the teichoic acid-like Lancefield group B carbohydrate (GBC) and type-specific capsular

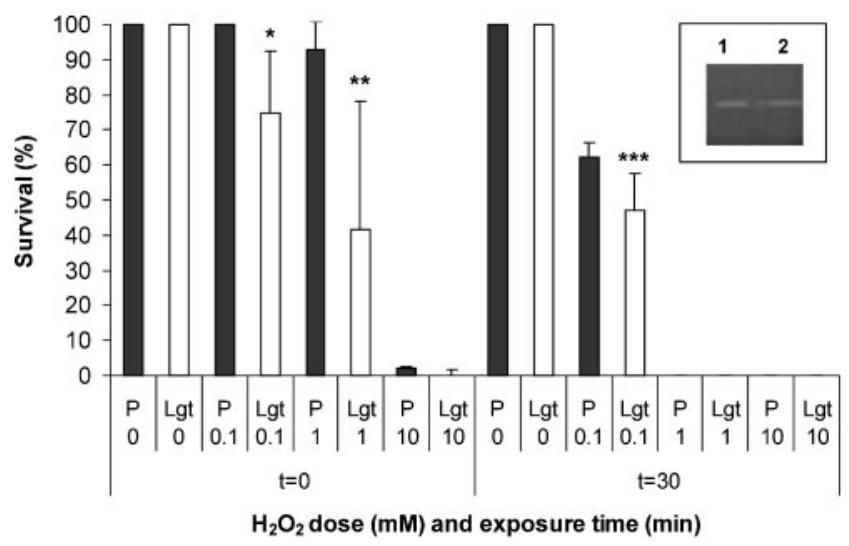

Fig. 3. Sensitivity of $S$. agalactiae $A 909$ and A909 $\Delta$ Lgt to oxidative stress, as determined by challenge with hydrogen peroxide. The doses shown are the concentrations (mM) of hydrogen peroxide added to cultures. Culture samples were taken immediately post-addition $(t=0)$ and after $30 \mathrm{~min}$ incubation $(t=30)$. Significance was assessed by Student's $t$-test between the parent $(P)$ and $\Delta \mathrm{Lgt}(\mathrm{Lgt})$ strains grown under the same conditions. *, $P=0.02 ;{ }^{* \star}, P=0.006 ;{ }^{* \star *}, P=0.046$. Inset: proteins extracted from the parent strain (lane 1) and the A909 $\Delta$ Lgt mutant (lane 2) were separated by native PAGE and superoxide dismutase activity was visualized zymographically. Each lane contains $20 \mu \mathrm{g}$ total protein. 
polysaccharides on their cell surfaces (Sutcliffe et al., 2008; Cieslewicz et al. 2005). The principal GBC biosynthesis locus contains a putative Lpp of unknown function (Sutcliffe et al., 2008). We therefore examined if Lgt mutation affected incorporation of the GBC into the cell wall of strain A909 $\Delta$ Lgt. The level of surface GBC was estimated by measuring the time taken for visible agglutination to be observed using a commercial diagnostic kit that reacts with GBC. As shown in Fig. 4, there was a statistically significant reduction in the speed at which A909 $\Delta$ Lgt cells were agglutinated, suggesting that lower amounts of the GBC are displayed on the cell surface.

To further investigate effects of Lgt mutation on the cell envelope, semiquantitative immunoblotting experiments were carried out with anti-capsular antibody. On the basis of the intensity of spots at different dilutions of bacterial cultures it appeared that there was an approximately twofold reduction in the level of type Ia capsular antigen in the A909 $\Delta$ Lgt strain compared with the parent strain (Fig. 5a). Electron microscopic analysis of the strains following immunogold labelling suggested that capsule coverage of the A909 $\Delta$ Lgt strain was patchier than in the parent strain (Fig. 5b). These data suggest a perturbation of cell envelope organization that may be an indirect consequence of the effect on GBC noted above.

\section{Effect of Lgt mutation on GBS endothelial cell adherence and invasion in vitro}

The complete GBS genomes encode at least two putative Lpp that may influence adhesion and cell invasion, i.e. the laminin binding protein Lmb and a paralogue (Spellerberg

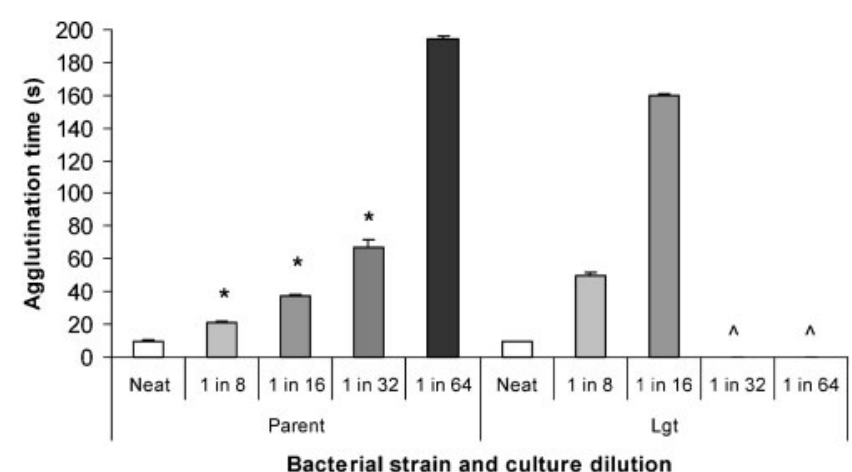

Fig. 4. Group $B$ carbohydrate (GBC) production by $S$. agalactiae A909 and A909 $\Delta$ Lgt. The ability of the $S$. agalactiae A909 (Parent) and A909 $\Delta$ Lgt (Lgt) strains to produce the GBC was evaluated by monitoring the time taken for antibody-coated latex beads to agglutinate in the presence of different dilutions of bacterial cultures. Significance was assessed by Student's $t$-test. * indicates a statistically significant difference in the time taken for agglutination to occur between the parent and $\Delta$ Lgt strains $(P<0.001) . \wedge$ indicates that no agglutination was visible within $15 \mathrm{~min}$ of application of latex beads. (a)

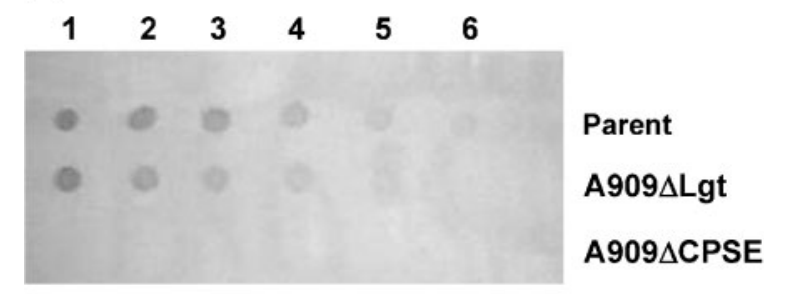

(b)

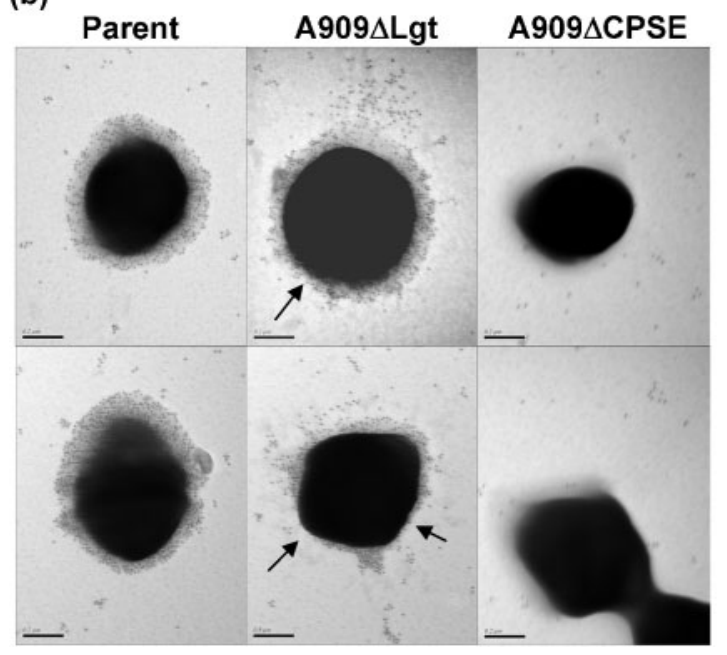

Fig. 5. Polysaccharide capsule production by S. agalactiae A909 and A909 L Lgt. (a) Capsular polysaccharide production by $S$. agalactiae A909, $\Delta \mathrm{Lgt}$ and $\Delta$ CPSE. Aliquots $(3 \mu \mathrm{l})$ of serial dilutions of bacterial cultures were spotted onto nitrocellulose and probed with rabbit-anti type la CPS antiserum to determine the level of capsule production. Column 1, neat culture $\left(\mathrm{OD}_{600} 1.0\right)$; column 2, 1 in 2 dilution; column 3, 1 in 4 dilution; column 4, 1 in 8 dilution; column 5, 1 in 16 dilution; column 6, 1 in 32 dilution. (b) Visualization of capsule by immunogold labelling. Preparations of the three $S$. agalactiae strains were probed with rabbit anti-type la CPS antiserum, which was then detected using anti-rabbit IgGconjugated gold particles. Samples were negatively stained to allow visualization by electron microscopy at $75000 \times$ magnification. Scale bars represent $0.2 \mu \mathrm{m}$. Arrows indicate regions of the bacterial cells significantly depleted of capsule in the A909 $\mathrm{Lgt}$ strain.

et al., 1999; Sutcliffe \& Harrington, 2004; Tenenbaum et al., 2007). The loss of Lgt activity in the A909 LLt strain would thus be predicted to influence adherence and/or invasion. To investigate this, the ability of GBS strains to adhere to and invade HUVEC-C cells in tissue culture was measured. Since umbilical vein endothelial cells are fetally derived they can be considered a relevant model for GBS adherence/invasion in the neonate. Although considerable inter-assay variation was observed (data not shown), the data from five biological replicates revealed that, compared to the parent strain, the A909ALgt strain exhibited a statistically significant $(P=0.0079)$ reduction in adherence 


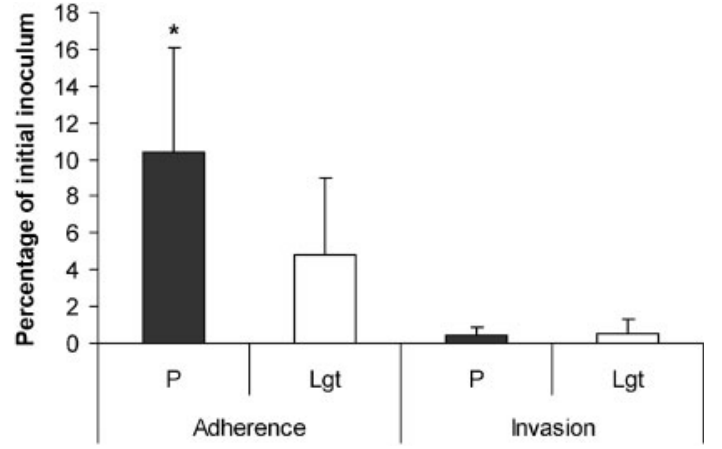

Fig. 6. Adherence to and invasion of endothelial cells by $S$. agalactiae A909 and A909 L Lgt. Adherence and invasion of HUVEC-C cells by S. agalactiae A909 (P) and A909 LLgt (Lgt) were measured by the method of Rubens et al. (1992). The data shown represent combined results from five individual experiments. Significance was assessed by Student's $t$-test. *, $P=0.0079$.

to HUVEC-C cells (Fig. 6) but no significant difference in cell invasion. These data strongly suggest that correctly localized Lpp are required for efficient GBS adherence to endothelial cells. The absence of a significant effect on invasion is surprising given recent data suggesting that $\mathrm{Lmb}$ is required for invasion of human brain microvascular endothelial cells (Tenenbaum et al., 2007), whilst several putative lipoproteins have been shown to be upregulated during in vitro growth conditions associated with highlevel invasion of cervical epithelial cells (Johri et al., 2007).

\section{Concluding comments}

This study has demonstrated that a viable GBS mutant in lgt can be generated in vitro and that the mutant fails to lipidate precursor Lpp (Fig. 1), resulting in their aberrant processing (Fig. 2). These data are in excellent agreement with the recent report by Henneke et al. (2008) of an Lgt mutant of GBS strain NEM316. Thus, the phenotype of strain A909 $\Delta$ Lgt would be expected to show pleiotropic changes associated with the loss or reduced efficiency of Lpp function. Surprisingly, many of the phenotypes assayed for A909 LLgt in this study showed no significant changes compared with the parent strain. Nevertheless there were clear changes in the cell envelope organization (Figs 4 and 5) and these correlated with a statistically significant reduction in the ability of strain A909 $\Delta$ Lgt to adhere to cultured endothelial cells (Fig. 6). Strain A909 $\Delta$ Lgt also showed an increased sensitivity to oxidative stress (Fig. 3), suggesting that it may be less able to resist the reactive oxygen intermediates unleashed by host phagocytes. These data are interesting in the context of the findings of Henneke et al. (2008), who observed that an Lgt deletion mutant of GBS strain NEM316 exhibited a 'hypervirulent' phenotype in a subcutaneous mouse infection model of GBS sepsis. It is notable that a Staphylococcus aureus Lgt mutant also exhibited a 'hyper- virulent' phenotype (Bubeck Wardenburg et al., 2006). It would appear that, even though virulence traits such as adhesion and resistance to oxidative stress may be compromised by the loss of specific Lpp, the inability to lipidate Lpp results in a failure to activate immune responses, notably those of the innate immune system (Stoll et al., 2005; Bubeck Wardenburg et al., 2006; Henneke et al., 2008). This immune evasion appears to contribute to a hypervirulence phenotype, at least with some Lgt-deficient strains in some animal models. In contrast, mutations inactivating lgt in other Gram-positive pathogens have not resulted in hypervirulent phenotypes and in some cases have led to attenuation (Petit et al., 2001; Hamilton et al., 2006; Baumgärtner et al., 2007; Machata et al., 2008). The data for GBS suggest that it may be prudent for future studies to focus on the roles of individual Lpp as potential virulence factors and vaccine candidates, particularly since the significant effects on adhesion reported here suggest that some Lpp may be important in neonatal colonization. However, for comparison, it would also be of interest to determine the virulence phenotypes of GBS $l s p$ and $\lg t / l s p$ mutants such as those described by Henneke et al. (2008).

\section{ACKNOWLEDGEMENTS}

We would like to thank Professor Jamie Leigh (University of Nottingham, UK), Dr Domenico Maoine (Chiron Corporation, Siena, Italy) and Dr Donald Chaffin (Children's Hospital and Medical Center, Seattle, USA) for provision of anti-MtuA, anti-SAG808 and anti-CPS (Ia) antisera, respectively. We are grateful to Dr Anne Graham (University of Bradford, UK) for providing HUVEC-C.

\section{REFERENCES}

Baumgärtner, M., Karst, U., Gerstel, B., Loessner, M., Wehland, J. \& Jansch, L. (2007). Inactivation of Lgt allows systematic characterization of lipoproteins from Listeria monocytogenes. J Bacteriol 189, 313324.

Biswas, I., Gruss, A., Ehrlich, S. D. \& Maguin, E. (1993). Highefficiency gene inactivation and replacement system for Grampositive bacteria. J Bacteriol 175, 3628-3635.

Braun, V. \& Wu, H. C. (1994). Lipoproteins: structure-function, biosynthesis and model for protein export. New Comp Biochem 27, 319-341.

Bray, B. A., Sutcliffe, I. C. \& Harrington, D. J. (2009). Expression of the MtsA lipoprotein of Streptococcus agalactiae A909 is regulated by manganese and iron. Antonie Van Leeuwenhoek 95, 101-109.

Brodeur, B. R., Boyer, M., Charlebois, I., Hamel, J., Couture, F., Rioux, C. R. \& Martin, D. (2000). Identification of group B streptococcal Sip protein, which elicits cross-protective immunity. Infect Immun 68, 5610-5618.

Bubeck Wardenburg, J., Williams, W. A. \& Missiakas, D. (2006). Host defenses against Staphylococcus aureus infection require recognition of bacterial lipoproteins. Proc Natl Acad Sci U S A 103, 13831-13836.

Chaffin, D. O., Beres, S. B., Yim, H. H. \& Rubens, C. E. (2000). The serotype of type Ia and III group B streptococci is determined by the polymerase gene within the polycistronic capsule operon. J Bacteriol 182, 4466-4477. 
Cieslewicz, M. J., Chaffin, D., Glusman, G., Kasper, D., Madan, A., Rodrigues, S., Fahey, J., Wessels, M. R. \& Rubens, C. E. (2005). Structural and genetic diversity of Group B Streptococcus capsular polysaccharides. Infect Immun 73, 3096-3103.

Denham, E. L., Ward, P. N. \& Leigh, J. A. (2008). Lipoprotein signal peptides are processed by Lsp and Eep of Streptococcus uberis. J Bacteriol 190, 4641-4647.

Denham, E. L., Ward, P. N. \& Leigh, J. A. (2009). In the absence of Lgt, lipoproteins are shed from Streptococcus uberis independently of Lsp. Microbiology 155, 134-141.

Dixon, S., Haswell, M., Harrington, D. \& Sutcliffe, I. C. (2001). Surface immunolocalisation of HPr in the equine pathogen Streptococcus equi. Syst Appl Microbiol 24, 486-489.

Glaser, P., Rusniok, C., Buchrieser, C., Chevalier, F., Frangeul, L., Msadek, T., Zouine, M., Couve, E., Lalioui, L. \& other authors (2002). Genome sequence of Streptococcus agalactiae, a pathogen causing invasive neonatal disease. Mol Microbiol 45, 1499-1513.

Hamilton, A., Harrington, D. J. \& Sutcliffe, I. C. (2000). Characterization of acid phosphatase activities in the equine pathogen Streptococcus equi. Syst Appl Microbiol 23, 325-329.

Hamilton, A., Robinson, C., Sutcliffe, I. C., Slater, J., Maskell, D. J., Davis-Poynter, N., Smith, K., Waller, A. \& Harrington, D. J. (2006). Mutation of the maturase lipoprotein attenuates the virulence of Streptococcus equi to a greater extent than does loss of general lipoprotein lipidation. Infect Immun 74, 6907-6919.

Heath, P. T., Balfour, G., Weisner, A. M., Esfratiou, A., Lamagni, T. L., Tighe, H., O'Connell, L. A. F., Cafferkey, M., Verlander, N. Q. \& other authors (2004). Group B streptococcal disease in UK and Irish infants younger than 90 days. Lancet 363, 292-294.

Henneke, P. \& Berner, R. (2006). Interaction of neonatal phagocytes with group B Streptococcus: recognition and response. Infect Immun 74, 3085-3095.

Henneke, P., Dramsi, S., Mancuso, G., Chraibi, K., Pellegrini, E., Theilacker, C., Hubner, J., Santos-Sierra, S., Teti, G. \& other authors (2008). Lipoproteins are critical TLR2 activating toxins in group B streptococcal sepsis. J Immunol 180, 6149-6158.

Hutchings, M. I., Palmer, T., Harrington, D. J. \& Sutcliffe, I. C. (2009). Lipoprotein biogenesis in Gram-positive bacteria: knowing when to hold 'em, knowing when to fold 'em. Trends Microbiol 17, 13-21.

Janulczyk, R., Ricci, S. \& Björck, L. (2003). MtsABC is important for manganese, iron transport, oxidative stress resistance and virulence of Streptococcus pyogenes. Infect Immun 71, 2656-2664.

Johnston, J. W., Myers, L. E., Ochs, M. M., Benjamin, W. H., Jr, Briles, D. E. \& Hollingshead, S. K. (2004). Lipoprotein PsaA in virulence of Streptococcus pneumoniae: surface accessibility, role in protection from superoxide. Infect Immun 72, 5858-5867.

Johri, A. K., Paoletti, L. C., Glaser, P., Dua, M., Sharma, P. K., Grandi, G. \& Rappuoli, R. (2006). Group B Streptococcus: global incidence and vaccine development. Nat Rev Microbiol 4, 932-942.

Johri, A. K., Margarit, I., Broenstrup, M., Brettoni, C., Hua, L., Gygi, S. P., Telford, J. L., Grandi, G. \& Paoletti, L. C. (2007). Transcriptional and proteomic profiles of group B Streptococcus type V reveal potential adherence proteins associated with high-level invasion. Infect Immun 75, 1473-1483.

Leskelä, S., Wahlstron, E., Kontinen, V. P. \& Sarvas, M. (1999). Lipid modification of prelipoproteins is dispensable for growth but essential for efficient protein secretion in Bacillus subtilis: characterization of the lgt gene. Mol Microbiol 31, 1075-1085.

Low, Y. L., Jakubovics, N. S., Flatman, J. C., Jenkinson, H. F. \& Smith, A. W. (2003). Manganese-dependent regulation of the endocarditis- associated virulence factor EfaA of Enterococcus faecalis. J Med Microbiol 52, 113-119.

Machata, S., Tchatalbachev, S., Mohamed, W., Jänsch, L., Hain, T. \& Chakraboty, T. (2008). Lipoproteins of Listeria monocytogenes are critical for virulence and TLR2-mediated immune activation. J Immunol 181, 2028-2035.

Maguin, E., Prevost, H., Ehrlich, S. D. \& Gruss, A. (1996). Efficient insertional mutagenesis in lactococci and other Gram-positive bacteria. J Bacteriol 178, 931-935.

Petit, C. M., Brown, J. R., Ingraham, K., Bryant, A. P. \& Holmes, D. J. (2001). Lipid modification of prelipoproteins is dispensable for growth in vitro but essential for virulence in Streptococcus pneumoniae. FEMS Microbiol Lett 200, 229-233.

Rahman, O., Cummings, S. P., Harrington, D. J. \& Sutcliffe, I. C. (2008). Methods for the bioinformatic identification of bacterial lipoproteins encoded in the genomes of Gram-positive bacteria. World J Microbiol Biotechnol 24, 2377-2382.

Rubens, C. E., Smith, S., Hulse, M., Chi, E. Y. \& van Belle, G. (1992). Respiratory epithelial cell invasion by group B streptococci. Infect Immun 60, 5157-5163.

Smith, A. J., Ward, P. N., Field, T. R., Jones, C. L., Lincoln, R. A. \& Leigh, J. A. (2003). MtuA, a lipoprotein receptor antigen from Streptococcus uberis is responsible for acquisition of manganese during growth in milk, is essential for infection of the lactating bovine mammary gland. Infect Immun 71, 4842-4849.

Spellerberg, B., Rozdzinski, E., Martin, S., Weber-Heynemann, J., Schnitzler, N., Lutticken, R. \& Podbielski, A. (1999). Lmb, a protein with similarities to the LraI adhesin family, mediates attachment of Streptococcus agalactiae to human laminin. Infect Immun 67, 871-878.

Stoll, H., Dengjel, J., Nerz, C. \& Götz, F. (2005). Staphylococcus aureus deficient in lipidation of prelipoproteins is attenuated in growth and immune activation. Infect Immun 73, 2411-2423.

Sutcliffe, I. C. \& Harrington, D. J. (2002). Pattern searches for the identification of putative lipoprotein genes in Gram positive bacterial genomes. Microbiology 148, 2065-2077.

Sutcliffe, I. C. \& Harrington, D. J. (2004). Putative lipoproteins of Streptococcus agalactiae identified by bioinformatic genome analysis. Antonie Van Leeuwenhoek 85, 305-315.

Sutcliffe, I. C. \& Russell, R. R. B. (1995). Lipoproteins of Grampositive bacteria. J Bacteriol 177, 1123-1128.

Sutcliffe, I. C., Black, G. \& Harrington, D. J. (2008). Bioinformatic insights into the biosynthesis of the group B carbohydrate in Streptococcus agalactiae. Microbiology 154, 1354-1363.

Tenenbaum, T., Spellerberg, B., Adam, R., Vogel, M., Kim, K. S. \& Schroten, H. (2007). Streptococcus agalactiae invasion of human brain microvascular endothelial cells is promoted by the laminin-binding protein Lmb. Microbes Infect 9, 714-720.

Tettelin, H., Masignani, V., Cieslewicz, M. J., Eisen, J. A., Peterson, S., Wessels, M. R., Paulsen, I. T., Nelson, K. E., Margarit, I. \& other authors (2002). Complete genome sequence and comparative genomic analysis of an emerging human pathogen, serotype $\mathrm{V}$ Streptococcus agalactiae. Proc Natl Acad Sci U S A 99, 12391-12396.

Tettelin, H., Masignani, V., Cieslewicz, M. J., Donati, C., Medini, D., Ward, N. L., Angiuoli, S. V., Crabtree, J., Jones, A. L. \& other authors (2005). Genome analysis of multiple pathogenic isolates of Streptococcus agalactiae: implications for the microbial "pangenome". Proc Natl Acad Sci U S A 102, 13950-13955.

Edited by: M. Kilian 